\title{
Exact Height Distributions for the KPZ Equation with Narrow Wedge Initial Condition
}

\author{
Tomohiro Sasamoto ${ }^{\star}$ and Herbert Spohn ${ }^{\dagger}$ \\ * Department of Mathematics and Informatics, Chiba University, \\ 1-33 Yayoi-cho, Inage, Chiba 263-8522, Japan \\ e-mail: sasamoto@math.s.chiba-u.ac.jp \\ $\dagger$ Zentrum Mathematik and Physik Department, TU München, \\ D-85747 Garching, Germany \\ e-mail: spohn@ma.tum.de
}

\begin{abstract}
We consider the KPZ equation in one space dimension with narrow wedge initial condition, $h(x, t=0)=-|x| / \delta, \delta \ll 1$. Based on previous results for the weakly asymmetric simple exclusion process with step initial conditions, we obtain a determinantal formula for the one-point distribution of the solution $h(x, t)$ valid for any $x$ and $t>0$. The corresponding distribution function converges in the long time limit, $t \rightarrow \infty$, to the Tracy-Widom distribution. The first order correction is a shift of order $t^{-1 / 3}$. We provide numerical computations based on the exact formula.
\end{abstract}




\section{Introduction}

In a seminal paper [1] Kardar, Parisi, and Zhang (KPZ) proposed a continuum equation for surface growth, which in the particular case of one space-dimension reads

$$
\frac{\partial}{\partial t} h=\frac{1}{2} \lambda\left(\frac{\partial}{\partial x} h\right)^{2}+\nu \frac{\partial^{2}}{\partial x^{2}} h+\sqrt{D} \eta
$$

Here $h(x, t)$ is the height profile at time $t, t \geq 0$, and $x \in \mathbb{R} . \lambda$ is the strength of the nonlinearity. (1.1) is invariant under $\lambda \rightsquigarrow-\lambda$ and $h \rightsquigarrow-h$. For later convenience we choose $\lambda>0 . \nu>0$ is the coefficient of diffusive relaxation, $\eta$ normalized Gaussian white noise with covariance $\left\langle\eta(x, t) \eta\left(x^{\prime}, t^{\prime}\right)\right\rangle=\delta\left(x-x^{\prime}\right) \delta\left(t-t^{\prime}\right)$, and $\sqrt{D}$ the noise strength. (1.1) is the KPZ equation in one space dimension.

Typical realizations of the white noise $\eta(x, t)$ are far from regular functions. The solution $h(x, t)$ partially inherits this roughness of the noise and therefore the square $(\partial h / \partial x)^{2}$ is ill-defined, in general. Nevertheless meaningful solutions can be constructed by suitable approximation schemes. They will be explained in detail below. The most direct one can be easily stated. One smoothens $\eta$ to $\eta_{\kappa}$ as

$$
\eta_{\kappa}(x, t)=\int \mathrm{d} x^{\prime} \kappa \varphi\left(\kappa\left(x-x^{\prime}\right)\right) \eta\left(x^{\prime}, t\right)=\varphi_{\kappa} * \eta(x, t)
$$

with $\varphi$ some smooth, localized, and normalized smearing function. Then $\eta_{\kappa} \rightarrow \eta$ as $\kappa \rightarrow \infty$. (1.1) with noise $\eta_{\kappa}$ has well-defined solutions, denoted by $h_{\kappa}(x, t)$. They move with a uniform background velocity $v_{\kappa}$ along the $h$-direction. $v_{\kappa} \rightarrow \infty$ as $\kappa \rightarrow \infty$, but $h_{\kappa}(x, t)-v_{\kappa} t$ has a limit. Since $v_{\kappa}$ sets merely the choice of a reference frame, the claim is that under this limit procedure the fluctuation properties remain intact.

While meaningful solutions are thus ensured, very little is known about their properties. To make $\partial h(x, t) / \partial x$ stationary, one has to start the KPZ equation with two-sided Brownian motion. With this input, one argues that the height fluctuations will grow as $t^{1 / 3}$, while the transverse correlation length increases as $t^{2 / 3}$. Very recently it has been proved that the variance of the stationary two-point function increases as $t^{4 / 3}$ by providing suitable upper and lower bounds $[2]$.

In our investigation we choose as initial data the narrow wedge

$$
h(x, 0)=-|x| / \delta
$$

with $\delta \ll 1$. This may look artificial. However, for short times the nonlinearity dominates and, ignoring the other terms in the equation, $h$ spreads very rapidly into the parabolic profile

$$
h(x, t)= \begin{cases}-x^{2} / 2 \lambda t & \text { for }|x| \leq 2 \lambda t / \delta \\ -|x| / \delta & \text { for }|x|>2 \lambda t / \delta\end{cases}
$$

which should be viewed as the top part of a growing droplet. Physically one thereby covers the case of macroscopically curved height profiles. 
We will provide a determinantal formula for the one-point distribution of $h(x, t)$ at prescribed $x$ and valid for all $t>0$, which is exact in the properly normalized limit $\delta \rightarrow 0$. The formula will be given in Equation (4.17). Numerical plots based on this formula are provided in Section 6.

The exact solution is constructed from the corner growth model, which we introduce first. The corner growth is the stochastic evolution of a height function $h(j, t)$, $j \in \mathbb{Z}, t \geq 0$, which takes integer values constrained by $|h(j+1, t)-h(j, t)|=1$. The initial condition is the wedge $h(j, 0)=-|j|$. In a short time interval $\mathrm{d} t$, independently for each height, at a local minimum the height function increases by 2 with probability $p \mathrm{~d} t$ and stays put with probability $1-p \mathrm{~d} t$. Correspondingly, at a local maximum it decreases by 2 with probability $q \mathrm{~d} t$ and stays put with probability $1-q \mathrm{~d} t$. These rules respect the constraint. We set $q+p=1$ and $0 \leq p<q \leq 1$, so the height will decrease on average. It has been proved in [4, 5], see also [6] for the case $p=0$, that

$$
h(0, t) \cong-\frac{1}{2}(q-p) t+2^{-1 / 3}((q-p) t)^{1 / 3} \xi_{\mathrm{TW}}
$$

for large t. $1 / 3$ is the scaling exponent predicted already on the basis of the KPZ equation 1.1. $\xi_{\mathrm{TW}}$ is a random amplitude which is Tracy-Widom distributed, i.e.

$$
\mathbb{P}\left(\xi_{\mathrm{TW}} \leq s\right)=F_{\mathrm{TW}}(s)=\operatorname{det}\left(1-P_{s} K_{\mathrm{Ai}} P_{s}\right)
$$

with $K_{\mathrm{Ai}}$ the Airy kernel,

$$
K_{\mathrm{Ai}}(x, y)=\int_{0}^{\infty} \mathrm{d} \lambda \operatorname{Ai}(x+\lambda) \operatorname{Ai}(y+\lambda),
$$

Ai the Airy function, and with $P_{s}$ the projection onto the interval $[s, \infty) . P_{s} K_{\mathrm{Ai}} P_{s}$ is a trace class operator on $L^{2}(\mathbb{R})$. Hence the Fredholm determinant $(1.6)$ is welldefined on $L^{2}(\mathbb{R})$.

As will be discussed in detail, the exact KPZ solution is obtained through the corner growth model in the limit of small asymmetry, more precisely one requires $q-p \ll 1$, space of order $(q-p)^{-2}$, and time of order $(q-p)^{-4}$. For large $t$ the exact solution attains the Tracy-Widom statistics as in (1.5). The solution also predicts that the first order correction to Tracy-Widom is a shift of order $t^{-1 / 3}$. Such qualitative features remain valid over a much wider range of $q-p$ than anticipated by the method of construction [3]. For the corner growth model the maximal asymmetry is $q=1$. We expect that upon decreasing $q$ towards $1 / 2$ finer and finer properties of the exact solution are revealed. At present such claims can be tested only through Monte Carlo simulations. For example, at $q=1$ for $t=10^{3} \mathrm{MC}$ steps the average height is -512 and its distribution has an effective range of 30 steps each of size 2 . Even at this discrete level the Tracy-Widom distribution is an accurate approximation provided one includes a shift of 2.9 to the right relative to the approximation of 1.5. For smaller values of $q$, approximately at $q=0.78$, the prefactor of the $t^{-1 / 3}$ shift changes sign, which again can be understood from the KPZ solution. To leading 
order in $t$ the solution is shifted by $\gamma_{t}^{-1}(-0.577+2 \log (q-p)), \gamma_{t}=2^{-1 / 3}(q-p)^{4 / 3} t^{1 / 3}$, which is indeed negative. To study finer details would require longer Monte Carlo runs, which have not been carried out yet. Available is the $t^{-1 / 3}$ correction of the Tracy-Widom distribution for the polynuclear growth model, as based on an exact recursion formula [7]. For large $t$ a definite limit is approached. The correction has a single node. Thus it agrees qualitatively with the $t^{-1 / 3}$ correction of our solution, which is proportional to $F_{\mathrm{TW}}^{\prime \prime}(s)$, but the precise shape of the correction is different. Apparently the first order correction to Tracy-Widom depends on the details of the model.

\section{The Cole-Hopf solution}

We start from the mollified KPZ equation

$$
\frac{\partial}{\partial t} h_{\kappa}=\frac{1}{2} \lambda\left(\frac{\partial}{\partial x} h_{\kappa}\right)^{2}+\nu \frac{\partial^{2}}{\partial x^{2}} h_{\kappa}+\sqrt{D} \eta_{\kappa}
$$

where the Gaussian noise $\eta_{\kappa}$ is smeared as in $(1.2)$ and hence has the covariance

$$
\left\langle\eta_{\kappa}(x, t) \eta_{\kappa}\left(x^{\prime}, t^{\prime}\right)\right\rangle=\varphi_{\kappa} * \varphi_{\kappa}\left(x-x^{\prime}\right) \delta\left(t-t^{\prime}\right) \text {. }
$$

The mollifier $\varphi_{\kappa}$ satisfies $\varphi_{\kappa}(x)=\kappa \varphi(\kappa x), \varphi \geq 0, \varphi$ of rapid decay, $\varphi(x)=\varphi(-x)$, and $\int \varphi(x) \mathrm{d} x=1$. The average over space-time white noise is denoted by $\langle\cdot\rangle \cdot(2.1)$ becomes linear through the Cole-Hopf transformation

$$
Z_{\kappa}(x, t)=\exp \left[(\lambda / 2 \nu) h_{\kappa}(x, t)\right]
$$

in which case $Z_{\kappa}$ satisfies

$$
\frac{\partial}{\partial t} Z_{\kappa}=\nu \frac{\partial^{2}}{\partial x^{2}} Z_{\kappa}+(\lambda \sqrt{D} / 2 \nu) \eta_{\kappa} Z_{\kappa}
$$

The solution to 2.4 can be written in terms of the Feynman-Kac formula by introducing an auxiliary standard Brownian motion $b(t)$ with variance $t$. Denoting $\mathbb{E}_{x}$ its expectation when starting at $x$, one has

$$
Z_{\kappa}(x, t)=\mathbb{E}_{x}\left(\exp \left[(\lambda \sqrt{D} / 2 \nu) \int_{0}^{t} \eta_{\kappa}(b(2 \nu s), t-s) \mathrm{d} s\right] Z(b(2 \nu t), 0)\right)
$$

for some deterministic initial condition $Z(\cdot, 0)$.

In our context, the initial condition is a narrow wedge of the form 1.4 with a global off-set. More precisely we assume $h(x, 0)=(2 \nu / \lambda)\left(-\delta^{-1}|x|-\log 2 \delta\right), \delta \ll 1$. Then the Cole-Hopf transform $Z(x, 0)=(2 \delta)^{-1} \exp [-|x| / \delta]$ which is sharply peaked at $x=0$ and, as an idealization, we will set

$$
Z(x, 0)=\delta(x) .
$$


Note that the average

$$
\left\langle Z_{\kappa}(x, t)\right\rangle=\exp \left[\frac{1}{2}(\lambda \sqrt{D} / 2 \nu)^{2} \varphi_{\kappa} * \varphi_{\kappa}(0) t\right] \mathbb{E}_{x}(Z(b(2 \nu t), 0)) .
$$

Since $\varphi_{\kappa} * \varphi_{\kappa}(0) \simeq \kappa$, it diverges as $\exp [c \kappa]$ with a suitable constant $c>0$. Thus to have a limit as $\kappa \rightarrow \infty$, one better divides the right hand side of (2.5) by $\left\langle Z_{\kappa}(x, t)\right\rangle$, which corresponds to the Wick ordering of the exponential, see for example [10]. Hence we define

$$
: Z_{\kappa}(x, t):=Z_{\kappa}(x, t) \exp \left[-\frac{1}{2}(\lambda \sqrt{D} / 2 \nu)^{2} \varphi_{\kappa} * \varphi_{\kappa}(0) t\right] .
$$

It is proved in [9] that, for continuous $Z(\cdot, 0)$ with an explicit decay condition for $|x| \rightarrow \infty$, the limit

$$
\lim _{\kappa \rightarrow \infty}: Z_{\kappa}(x, t):=Z(x, t)
$$

exists. $Z(x, t)$ is a well-defined stochastic process. With probability one it has sample paths which are continuous in both $x, t$. In addition $Z(x, t)>0$ provided $Z(x, 0) \geq 0$ and $Z(\cdot, 0) \neq 0$. With this input we define

$$
h(x, t)=\frac{2 \nu}{\lambda} \log Z(x, t)
$$

as the solution of the KPZ equation with $\delta$-correlated noise. For later purposes it is convenient to introduce, for arbitrary $\alpha>0$,

$$
\alpha h_{\alpha}(x, t)=\log \mathbb{E}_{x}\left(: \exp \left[\alpha \int_{0}^{t} \mathrm{~d} s \eta(b(s), t-s)\right]: \delta(b(t))\right) .
$$

Clearly $h_{\alpha}$ is the solution to the KPZ equation with $D=1$ and $\alpha=\lambda / 2 \nu$. By construction the first exponential moment is centered as

$$
\left\langle\mathrm{e}^{\alpha h_{\alpha}(x, t)}\right\rangle=(2 \pi t)^{-1 / 2} \mathrm{e}^{-\left(x^{2} / 2 t\right)} .
$$

From the point of view of the height function, the subtraction corresponds to

$$
h_{\kappa}(x, t)-\frac{1}{2}(\lambda D / 2 \nu) \varphi_{\kappa} * \varphi_{\kappa}(0) t .
$$

Hence the uniform velocity, alluded to in the introduction, is given by

$$
v_{\kappa}=\frac{1}{2}(\lambda D / 2 \nu) \varphi_{\kappa} * \varphi_{\kappa}(0) .
$$

(2.5) can be viewed also as a directed polymer subject to a space-time white noise random potential. With the initial condition $(2.6)$ the polymer $b(s)$ starts at $x$ and ends at 0 at time $2 \nu t$, which corresponds to a point-to-point directed polymer. The potential of the random environment is somewhat singular, hence the subtraction. $Z(x, t)$ is the random partition function of the directed polymer at the "inverse temperature" $\lambda \sqrt{D} / 2 \nu$. From the perspective of disordered systems, of interest is 
the random free energy $(2 \nu / \lambda) \log Z(x, t)$, which is nothing else than the KPZ height $h(x, t)$.

The observation leading to (2.7) extends to higher moments. We consider the $n$-th moment of $: Z_{\kappa}(t)$ : and for this purpose introduce $n$ independent standard Brownian motions $b_{j}(t), j=1, \ldots, n$, as replicas of $b(t)$. Then

$$
\begin{gathered}
\left\langle\left(: Z_{\kappa}(x, t):\right)^{n}\right\rangle=\mathbb{E}_{x} \times \ldots \times \mathbb{E}_{x}\left(\left\langle\operatorname { e x p } \left[(\lambda \sqrt{D} / 2 \nu) \sum_{j=1}^{n} \int_{0}^{t} \eta_{\kappa}\left(b_{j}(2 \nu s), t-s\right) \mathrm{d} s\right.\right.\right. \\
\left.\left.\left.-\frac{1}{2} n(\lambda \sqrt{D} / 2 \nu)^{2} \varphi_{\kappa} * \varphi_{\kappa}(0) t\right]\right\rangle \prod_{j=1}^{n} Z\left(b_{j}(2 \nu t), 0\right)\right) \\
=\mathbb{E}_{x} \times \ldots \times \mathbb{E}_{x}\left(\exp \left[\frac{1}{2}(\lambda \sqrt{D} / 2 \nu)^{2} \sum_{i, j=1, i \neq j}^{n} \int_{0}^{t} \varphi_{\kappa} * \varphi_{\kappa}\left(b_{i}(2 \nu s)-b_{j}(2 \nu s)\right) \mathrm{d} s\right]\right. \\
\left.\times \prod_{j=1}^{n} Z\left(b_{j}(2 \nu t), 0\right)\right),
\end{gathered}
$$

which is the path integral for $n$ quantum particles with pair potential $-\varphi_{\kappa} * \varphi_{\kappa}$. Thus the Wick ordering of the exponential in $(2.9)$ is identical to the normal ordering of the pair interaction. $\varphi_{\kappa}(x) \rightarrow \delta(x)$ as $\kappa \rightarrow \infty$ and 2.15) converges to the path integral of a system of $n$ quantum particles on the line interacting through an attractive $\delta$-potential. The corresponding hamiltonian reads

$$
H_{n}=-\sum_{j=1}^{n} \frac{1}{2} \frac{\partial^{2}}{\partial x_{j}^{2}}-\frac{1}{2} \alpha^{2} \sum_{i, j=1, i \neq j}^{n} \delta\left(x_{i}-x_{j}\right),
$$

where

$$
\alpha=(2 \nu)^{-3 / 2} \lambda D^{1 / 2} .
$$

Since $\mathrm{e}^{-t 2 \nu H_{n}}$ has a continuous kernel, it is a meaningful limit to set $Z(x, 0)=\delta(x)$ in 2.15 . Let us denote the $n$-particle state $\prod_{j=1}^{n} \delta\left(x-x_{j}\right)$ by $|x\rangle$. Then

$$
\left\langle Z(x, t)^{n}\right\rangle=\left\langle 0\left|\mathrm{e}^{-t 2 \nu H_{n}}\right| x\right\rangle
$$

with inner product in $L^{2}\left(\mathbb{R}^{n}\right)$.

For later purposes we record that, for the initial condition (2.6),

$$
\left\langle: Z_{\kappa}(x, t / 2 \nu):\right\rangle=\frac{1}{\sqrt{2 \pi t}} \exp \left[-x^{2} / 2 t\right]
$$

and hence also in the limit $\kappa \rightarrow \infty$, in agreement with 2.18 for $n=1$.

Very recently, Balaźs, Quastel, and Seppäläinen [2] studied the KPZ equation with initial condition $Z(x, 0)=\exp [(\lambda / 2 \nu) \tilde{b}(x)]$, where $\tilde{b}(x)$ is two-sided Brownian 
motion. They consider the solution as defined in 2.10 and prove, amongst other related results, upper and lower bounds of the form

$$
c_{-} t^{2 / 3} \leq \operatorname{Var}(h(x, t)) \leq c_{+} t^{2 / 3}
$$

We cannot treat their case, because we miss the yet to be accomplished extension of the contour integration formula in [4] to initial conditions given by the Bernoulli $\frac{1}{2}$ measure, see [8] for recent progress.

\section{The WASEP limit}

Bertini and Giacomin [9] (for short BG) discovered a rather different, and in a way physically better motivated, limiting procedure for the construction of solutions to the KPZ equation. They consider the weakly asymmetric version of the simple exclusion process (WASEP), which is equivalent to the corner growth model alluded to already in the introduction. In the height picture this corresponds to the following stochastic evolution: A height function $h: \mathbb{Z} \rightarrow \mathbb{Z}$ has to satisfy the constraint

$$
|h(j+1)-h(j)|=1
$$

for all $j \in \mathbb{Z}$. Independently, each height variable waits a unit exponentially distributed time. Then $h(j) \rightsquigarrow h(j)-2$ with probability $q$ and $h(j) \rightsquigarrow h(j)+2$ with probability $p, p+q=1$. Either transition is suppressed in case (3.1) is violated. The height differences are governed by the partially asymmetric simple exclusion process (PASEP). Here, because of (3.1), there is at most one particle per site and, under the exclusion constraint, particles jump with rate $p$ to the right and rate $q$ to the left. We choose $q>p$. Thus $h$ decreases to $-\infty$.

Weak asymmetry means that $q-p$ is small. The precise asymptotics can be studied in a scaling limit with dimensionless scale parameter $\varepsilon, \varepsilon \ll 1$. We choose a diffusive space-time scaling, i.e. time as $\varepsilon^{-2} t, t=\mathcal{O}(1)$, space as $\left\lfloor\varepsilon^{-1} x\right\rfloor, x \in \mathbb{R}$, $\lfloor\cdot\rfloor$ denoting integer part. The choice $q-p=\mathcal{O}(\varepsilon)$ is the widely studied WASEP, which has the deterministic KPZ equation $(D=0)$ as limit and Gaussian fluctuations relative to that profile [11]. In contrast to the conventional choice BG consider the crossover scale with an asymmetry equal to $\sqrt{\varepsilon}$, see [12] for a more complete discussion. For purpose of comparison with the KPZ equation we set more generally

$$
q-p=\beta \sqrt{\varepsilon}, \quad \beta>0 .
$$

For the initial height profile BG assume

$$
h(j, 0)=\left\lfloor\varepsilon^{-1 / 2} \phi(\varepsilon j)\right\rfloor,
$$

where $\phi$ is a smooth function with some decay condition at infinity. Here $\lfloor\cdot\rfloor$ means integer part subject to (3.1). We denote by $h^{\varepsilon}(j, t)$ the WASEP time-evolved height 
profile, the superscript $\varepsilon$ reminding that the rates are $\varepsilon$-dependent. BG prove the limit

$$
\lim _{\varepsilon \rightarrow 0} \sqrt{\varepsilon}\left(h^{\varepsilon}\left(\left\lfloor\varepsilon^{-1} x\right\rfloor, \varepsilon^{-2} t\right)+\frac{1}{2} t \varepsilon^{-3 / 2}\right)=h(x, t)+\frac{1}{24} t,
$$

where $h(x, t)$ is the solution of the KPZ equation, as defined in the previous section, with initial condition $\phi$ and parameters $\lambda=1, \nu=\frac{1}{2}, D=1$.

Note that $h(j+1,0)-h(j, 0)=\mathcal{O}(\sqrt{\varepsilon})$ and the particle density hence deviates by order $\sqrt{\varepsilon}$ from $\frac{1}{2}$. At that density the average time integrated particle current equals $-t \varepsilon^{-3 / 2} / 4$ to leading order, which is the subtraction on the left of (3.4). (There is a factor 2 when switching from integrated currents to heights.) The subtraction on the right is more subtle. As will be discussed in more detail below, it originates from the normalization condition

$$
\left\langle\mathrm{e}^{h(x, t)}\right\rangle=\int(2 \pi t)^{-1 / 2} \exp \left[-\left(x-x^{\prime}\right)^{2} / 2 t\right] \mathrm{e}^{\phi\left(x^{\prime}\right)} \mathrm{d} x^{\prime} .
$$

To implement for the WASEP height the narrow wedge initial condition from Section 2 , the natural choice would be density $\frac{1}{2}-\delta^{-1} \sqrt{\varepsilon}$ to the left and density $\frac{1}{2}+\delta^{-1} \sqrt{\varepsilon}$ to the right of the origin. Unfortunately, for such initial conditions there are no manageable formulas available and we have to take resort to initial conditions which no longer are covered by (3.4) and the results in [9].

As standing assumption, the WASEP has asymmetry $\beta \sqrt{\varepsilon}$ and an initial configuration such that all sites from 1 to $\infty$ are filled and all other sites are empty. The corresponding initial height profile is $h(j, 0)=-|j|$. After some short initial time span, the typical height profile becomes

$$
h^{\varepsilon}\left(j, \varepsilon^{-2} t\right) \cong \begin{cases}-\frac{1}{2}\left(j^{2} / \beta t\right) \varepsilon^{3 / 2}-\frac{1}{2} \beta t \varepsilon^{-3 / 2} & \text { for }|j| \leq \beta t \varepsilon^{-3 / 2} \\ -|j| & \text { for }|j|>\beta t \varepsilon^{-3 / 2}\end{cases}
$$

compare with (1.4). If one restricts $j$ to the interval $\left[-\varepsilon^{-1}, \varepsilon^{-1}\right]$, then there one has a parabolic profile, which scales exactly as assumed in (3.3) and thus supports that (3.4) remains valid for the initial sharp wedge. In 12 we obtained the one-point height distribution for the WASEP with 0-1 initial condition in the limit $\varepsilon \rightarrow 0$. Thus the plan is to use this result in the construction of the one-point distribution of the KPZ equation with narrow wedge initial condition.

Let us denote by $\eta_{j}(t)=0,1$ the occupation variables of the WASEP at site $j$ and time $t$. Since there is a random index $j_{0}$ such that $\eta_{j}(t)=0$ for $j<j_{0}$, one can define the WASEP height by

$$
h^{\varepsilon}(j, t)=-2 \sum_{\ell=-\infty}^{j} \eta_{\ell}(t)+j .
$$

Let $\mathcal{J}^{\varepsilon}(j, t)$ be the particle current across the bond $(j, j+1)$ integrated up to time $t$. Then

$$
h^{\varepsilon}(j, t)=2 \mathcal{J}^{\varepsilon}(j, t)-|j|
$$


In 12$]$ we studied the distribution of $\mathcal{J}^{\varepsilon}$ at $j=\left\lfloor y \beta t \varepsilon^{-3 / 2}+\varepsilon^{-1} x\right\rfloor$ and time $\varepsilon^{-2} t$. To identify the height statistics of the KPZ equation it suffices to take $y=0$, in which case

$$
\begin{gathered}
\lim _{\varepsilon \rightarrow 0} \mathbb{P}\left(\sqrt{\varepsilon} \beta h^{\varepsilon}\left(\left\lfloor\varepsilon^{-1} x\right\rfloor, \varepsilon^{-2} t\right)+\frac{1}{2} \beta^{2} t \varepsilon^{-1}+\left(x^{2} / 2 t\right)-\log (2 \beta \sqrt{\varepsilon}) \leq \gamma_{t} s\right) \\
=F_{t}(s),
\end{gathered}
$$

with

$$
\gamma_{t}=2^{-1 / 3}\left(\beta^{4} t\right)^{1 / 3}
$$

$F_{t}(s)$ is a $t$-dependent family of distribution functions. To define them, let us introduce the Airy kernel $K_{\mathrm{Ai}}(x, y)$ of (1.7), the kernel

$$
\begin{aligned}
B_{t}(x, y) & =K_{\mathrm{Ai}}(x, y) \\
+ & \int_{0}^{\infty} \mathrm{d} \lambda\left(\mathrm{e}^{\gamma_{t} \lambda}-1\right)^{-1}(\operatorname{Ai}(x+\lambda) \operatorname{Ai}(y+\lambda)-\operatorname{Ai}(x-\lambda) \operatorname{Ai}(y-\lambda)),
\end{aligned}
$$

and the unnormalized projection $P_{\mathrm{Ai}}$ with kernel

$$
P_{\mathrm{Ai}}(x, y)=\operatorname{Ai}(x) \operatorname{Ai}(y)
$$

Then

$$
\begin{aligned}
& F_{t}(s)=1-\int_{-\infty}^{\infty} \mathrm{d} u \exp \left[-\mathrm{e}^{\gamma_{t}(s-u)}\right] \\
& \times\left(\operatorname{det}\left(1-P_{u}\left(B_{t}-P_{\mathrm{Ai}}\right) P_{u}\right)-\operatorname{det}\left(1-P_{u} B_{t} P_{u}\right)\right) .
\end{aligned}
$$

Here $P_{u}$ projects onto $[u, \infty) . \quad P_{u} B_{t} P_{u}$ and $P_{u} P_{\mathrm{Ai}} P_{u}$ are trace class operators in $L^{2}(\mathbb{R})$. Hence the Fredholm determinants in $(3.13)$ are well-defined.

Note that the probability density $\frac{d}{d s} F_{t}(s)$ is the convolution of the Gumbel probability density $\gamma_{t} \mathrm{e}^{\gamma_{t} s} \exp \left[-\mathrm{e}^{\gamma_{t} s}\right]$ and the difference of determinants,

$$
g_{t}(u)=\operatorname{det}\left(1-P_{u}\left(B_{t}-P_{\mathrm{Ai}}\right) P_{u}\right)-\operatorname{det}\left(1-P_{u} B_{t} P_{u}\right) .
$$

For $t \rightarrow \infty$ the Gumbel density converges to $\delta(s)$ and $g_{t}(u)$ to $F_{\mathrm{TW}}^{\prime}(u)$, see [12]. Hence

$$
\lim _{t \rightarrow \infty} F_{t}(s)=F_{\mathrm{TW}}(s)
$$

with $F_{\mathrm{TW}}$ the Tracy-Widom distribution function 1.6 .

\section{Centering and exact solution}

Through the limit to $\delta$-correlated noise we adjusted the first moment of the partition function as

$$
\langle Z(x, t / 2 \nu)\rangle=\frac{1}{\sqrt{2 \pi t}} \exp \left[-\frac{x^{2}}{2 t}\right],
$$


which sets an important constraint for the WASEP limit. To exploit it, one notes that a particular exponential moment of the WASEP also satisfies a closed linear equation. This property was first proved by Gärtner [13, 14], see also [9] and the related contribution [15]. For the PASEP with fixed $p, q$ and arbitrary initial conditions, let us denote by $\mathbb{E}_{t}$ the expectation with respect to the height statistics at time $t$ and let us set

$$
f(j, t)=\mathbb{E}_{t}\left(\mathrm{e}^{\vartheta h(j)}\right) .
$$

$\vartheta$ is adjusted such that

$$
\mathrm{e}^{-2 \vartheta}=\frac{p}{q}
$$

Then $f$ is the solution of

$$
\begin{aligned}
\frac{d}{d t} f(j, t) & =\frac{1}{\cosh \vartheta}\left(\frac{1}{2} f(j+1, t)+\frac{1}{2} f(j-1, t)-f(j, t)\right)+\left(\frac{1}{\cosh \vartheta}-1\right) f(j, t), \\
f(j, 0) & =\mathrm{e}^{-\vartheta|j|} .
\end{aligned}
$$

(4.4) can be deduced by working out the time derivative as

$$
\frac{d}{d t} f(j, t)=\mathbb{E}_{t}\left(L \mathrm{e}^{\vartheta h(j)}\right)
$$

with $L$ the generator of the Markov jump process for the heights. To satisfy the constraint 4.1) one has to consider the height shifted by $\log f$ as

$$
\vartheta h^{\varepsilon}\left(\left\lfloor\varepsilon^{-1} x\right\rfloor, \varepsilon^{-2} t\right)-\log f\left(\left\lfloor\varepsilon^{-1} x\right\rfloor, \varepsilon^{-2} t\right)-\left(x^{2} / 2 t\right)-\log \sqrt{2 \pi t} .
$$

$f$ can be written as Fourier integral,

$$
\begin{aligned}
& f\left(\left\lfloor\varepsilon^{-1} x\right\rfloor, \varepsilon^{-2} t\right) \\
& =\frac{1}{2 \pi} \int_{-\pi}^{\pi} \mathrm{d} k \exp \left[(\cos k-1)(\cosh \vartheta)^{-1} \varepsilon^{-2} t+\mathrm{i} k \varepsilon^{-1} x\right. \\
& \left.\quad+\log \widehat{f}(k, 0)+\varepsilon^{-2} t\left((\cosh \vartheta)^{-1}-1\right)\right]
\end{aligned}
$$

with the initial condition

$$
\widehat{f}(k, 0)=\left(1-\mathrm{e}^{-2 \vartheta}\right)\left(1-2 \mathrm{e}^{-\vartheta} \cos k+\mathrm{e}^{-2 \vartheta}\right)^{-1} .
$$

$\log f\left(\left\lfloor\varepsilon^{-1} x\right\rfloor, \varepsilon^{-2} t\right)$ has to be computed including terms of order 1 . One has

$$
\begin{gathered}
\vartheta=\beta \varepsilon^{1 / 2}+\frac{1}{3} \beta^{3} \varepsilon^{3 / 2}+\mathcal{O}\left(\varepsilon^{5 / 2}\right), \\
(\cosh \vartheta)^{-1}=1-\frac{1}{2} \beta^{2} \varepsilon-\frac{1}{8} \beta^{4} \varepsilon^{2}+\mathcal{O}\left(\varepsilon^{3}\right) .
\end{gathered}
$$

The initial condition 4.8 is approximated by $2 / \beta \sqrt{\varepsilon}$. Then the Fourier integral is the transition probability of a simple random walk with rate $(\cosh \vartheta)^{-1}$ starting at 0 , which at the given scale can be approximated by a normalized Gaussian. Therefore

$$
\begin{aligned}
& -\log f\left(\left\lfloor\varepsilon^{-1} x\right\rfloor, \varepsilon^{-2} t\right) \\
& \quad=\frac{1}{2} \beta^{2} t \varepsilon^{-1}+\left(x^{2} / 2 t\right)+\frac{1}{8} \beta^{4} t+\log (\sqrt{2 \pi t} / \varepsilon)+\log (\beta \sqrt{\varepsilon} / 2)+\mathcal{O}(\sqrt{\varepsilon}) .
\end{aligned}
$$


Inserting in (4.6) and taking the deterministic approximation to $h^{\varepsilon}$ for the second order in the expansion of $\vartheta$, one arrives at

$$
\begin{aligned}
\vartheta h^{\varepsilon} & \left(\left\lfloor\varepsilon^{-1} x\right\rfloor, \varepsilon^{-2} t\right)-\log f\left(\left\lfloor\varepsilon^{-1} x\right\rfloor, \varepsilon^{-2} t\right)-\left(x^{2} / 2 t\right)-\log \sqrt{2 \pi t} \\
& =\beta \sqrt{\varepsilon} h^{\varepsilon}\left(\left\lfloor\varepsilon^{-1} x\right\rfloor, \varepsilon^{-2} t\right)+\frac{1}{2} \beta^{2} t \varepsilon^{-1}-\frac{1}{24} \beta^{4} t-\log (2 \sqrt{\varepsilon} / \beta)+\mathcal{O}(\sqrt{\varepsilon}) .
\end{aligned}
$$

Note that the $\varepsilon$-dependent part of the centering is precisely as in $(3.9)$.

For the WASEP at the reference point $y=0$ the average particle density $\rho$ equals $1 / 2$ and the noise strength equals $\rho(1-\rho)=1 / 4$. Including the factor 2 for the conversion between density and height differences, the corresponding parameters for the KPZ equation are $2 \nu=1$ and $D=1$. Since the asymmetry equals $\beta$, the WASEP height (4.12) should hence be compared with $\beta h_{\beta}(x, t)$, see (2.11). Then, stretching somewhat the results in [9], for the WASEP limit it holds

$$
\lim _{\varepsilon \rightarrow 0} \sqrt{\varepsilon} \beta h^{\varepsilon}\left(\left\lfloor\varepsilon^{-1} x\right\rfloor, \varepsilon^{-2} t\right)+\frac{1}{2} \beta^{2} t \varepsilon^{-1}-\frac{1}{24} \beta^{4} t-\log (2 \sqrt{\varepsilon} / \beta)=\beta h_{\beta}(x, t) .
$$

The $\mathrm{BG}$ result $(3.4)$ corresponds to the particular case $\beta=1$ and non-singular initial data.

Combining (4.13) and (3.9) one arrives at an exact formula for the one-point distribution of $h_{\beta}$ which reads

$$
\mathbb{P}\left(\beta h_{\beta}(x, t)+\frac{1}{24} \beta^{4} t+\left(x^{2} / 2 t\right)-2 \log \beta \leq \gamma_{t} s\right)=F_{t}(s) .
$$

To generalize to arbitrary $\lambda, \nu, D$ one starts from the definition 2.10 for the narrow wedge solution $h(x, t)$ of the KPZ equation and uses the scale invariance of white noise and of Brownian motion to obtain

$$
\begin{aligned}
(\lambda / 2 \nu) h(x, t / 2 \nu) & =\log \mathbb{E}_{x}\left(: \exp \left[(\lambda \sqrt{D} / 2 \nu) \int_{0}^{t / 2 \nu} \eta(b(2 \nu s),(t / 2 \nu)-s) \mathrm{d} s\right]: \delta(b(t))\right) \\
& =\log \mathbb{E}_{x}\left(: \exp \left[\left(\lambda \sqrt{D} /(2 \nu)^{3 / 2}\right) \int_{0}^{t} \eta(b(s), t-s) \mathrm{d} s\right]: \delta(b(t))\right) \\
& =\alpha h_{\alpha}(x, t)
\end{aligned}
$$

with $\alpha$ fixed to

$$
\alpha=(2 \nu)^{-3 / 2} \lambda D^{1 / 2} .
$$

Inserting in 4.14 one obtains the one-point distribution of the KPZ equation with narrow wedge initial data,

$$
\mathbb{P}\left((\lambda / 2 \nu) h(x, t / 2 \nu)+\frac{1}{12}\left(\gamma_{t}\right)^{3}+\left(x^{2} / 2 t\right)-2 \log \alpha \leq \gamma_{t} s\right)=F_{t}(s),
$$

where $\gamma_{t}=2^{-1 / 3}\left(\alpha^{4} t\right)^{1 / 3}$. The identity 4.17$)$ is the central result of our contribution.

Remark: After posting this article it was brought to our attention that G. Amir, I. Corwin, and J. Quastel independently obtained the identity (4.17) and the formula 3.13 for the distribution function $F_{t}$. 


\section{Some properties of the one-point distribution}

(i) Scale invariance. In terms of the original parameters we define $\alpha$ as in 4.16 and introduce dimensionless space, time, and height through

$$
X=\alpha^{2} x, \quad T=2 \nu \alpha^{4} t, \quad H=(\lambda / 2 \nu) h .
$$

Then the coefficients in the KPZ equation become $\lambda=1, \nu=\frac{1}{2}$, and $D=1$, i.e. $H(X, T)$ satisfies

$$
\frac{\partial}{\partial T} H=\frac{1}{2}\left(\frac{\partial H}{\partial X}\right)^{2}+\frac{1}{2} \frac{\partial^{2}}{\partial X^{2}} H+\eta .
$$

In these variables (4.17) reads

$$
\mathbb{P}\left(H(X, T)+\frac{1}{24} T+\left(X^{2} / 2 T\right)-2 \log \alpha \leq \tilde{\gamma}_{T} s\right)=F_{T}(s)
$$

with $\tilde{\gamma}_{T}=2^{-1 / 3} T^{1 / 3}$. The distribution function $F_{T}(s)$ depends on $T$ through substituting $\gamma_{t}$ by $\tilde{\gamma}_{T}$. The solution (4.17) satisfies the scale invariance of the KPZ equation, the term $2 \log \alpha$ resulting from the scaling of the initial condition $Z(x, 0)=\delta(x)$, see 2.11 .

(ii) Stationarity. Since for our initial conditions the height profile is curved, no stationarity in $x$ is expected. However, in the case of PNG droplet growth it was noted that height fluctuations become stationary once the systematic curvature is subtracted. The same feature appears for the KPZ equation. Using dimensionless units for simplicity, we set

$$
h(x, t)=\log \mathbb{E}_{0}\left(: \exp \left[\int_{0}^{t} \eta(b(s), s) \mathrm{d} s\right]: \delta(b(t)-x)\right),
$$

where, with no modification of the statistics, the Brownian motion $b(s)$ has been time-reversed to $b(t-s)$. Let us introduce the Brownian bridge $w(s)$ running from 0 at $s=0$ to $x$ at $s=t$. In terms of Brownian motion,

$$
w(s)=b(s)-\frac{s}{t}(b(t)-x) .
$$

Denoting by $\mathbb{E}_{0, x}^{\mathrm{BB}}$ the expectation over a Brownian bridge with endpoints $0, x$, we define

$$
\tilde{h}(x, t)=\log \mathbb{E}_{0, x}^{\mathrm{BB}}\left(: \exp \left[\int_{0}^{t} \eta(w(s), s) \mathrm{d} s\right]:\right),
$$

which satisfies the $x$-independent normalization

$$
\left\langle\mathrm{e}^{\tilde{h}(x, t)}\right\rangle=1
$$

Using scale invariance, one checks that $x \mapsto \tilde{h}(x, t)$ is stationary as a stochastic process for fixed $t$. On the level of the one-point distribution this property is reflected 
by $F_{t}(s)$ being independent of $x$.

(iii) Ground state energy of the $\delta$-Bose gas. As first shown by McGuire [16], in units of (2.18) with $2 \nu=1$ the ground state energy of the $\delta$-Bose gas with $n$ particles is given by

$$
E_{n}=-\frac{1}{24} n\left(n^{2}-1\right) \alpha^{4}
$$

By (2.18),

$$
\left\langle Z(0, t)^{n}\right\rangle=\left\langle 0\left|\mathrm{e}^{-t H_{n}}\right| 0\right\rangle \cong \mathrm{e}^{-t E_{n}}
$$

for large $t$. Of course, there are subleading terms, which however will be ignored at this stage. From the exact solution one has

$$
\mathbb{P}\left(\alpha h_{\alpha}(0, t)+\frac{1}{24} \alpha^{4} t-2 \log \alpha \leq s\right)=F_{t}\left(s / \gamma_{t}\right)
$$

Hence

$$
\begin{aligned}
\mathbb{E}(\exp & {\left.\left[n\left(\alpha h_{\alpha}(0, t)+\frac{1}{24} \alpha^{4} t-2 \log \alpha\right)\right]\right) } \\
= & \int \mathrm{d} s \int \mathrm{d} u \mathrm{e}^{n s} \mathrm{e}^{s} \mathrm{e}^{-\gamma_{t} u} \exp \left[-\mathrm{e}^{s} \mathrm{e}^{-\gamma_{t} u}\right] g_{t}(u) \\
= & n ! \int \mathrm{d} u \mathrm{e}^{n \gamma_{t} u} g_{t}(u)
\end{aligned}
$$

with $g_{t}(u)$ defined in 3.14 .

$\gamma_{t}$ diverges as $t^{1 / 3}$ for $t \rightarrow \infty$. Hence the integral in (5.11) is sensitive only to the right tail of $g_{t}(u)$. Furthermore, by the argument before (3.15), $g_{t}(u)$ tends to $F_{\mathrm{TW}}^{\prime}(u)$, which has a right tail as $\exp \left[-\frac{4}{3} u^{3 / 2}\right]$, see e.g. 17]. Therefore to leading order

$$
\int_{0}^{\infty} \mathrm{d} u \exp \left[-\frac{4}{3} u^{3 / 2}+n \gamma_{t} u\right] \cong \exp \left[\frac{1}{24} \alpha^{4} t n^{3}\right]
$$

for $t \rightarrow \infty$. Combining with 5.11,

$$
\lim _{t \rightarrow \infty}-\frac{1}{t} \log \mathbb{E}\left(\exp \left[n \alpha h_{\alpha}(0, t)\right]\right)=-\frac{1}{24} n\left(n^{2}-1\right) \alpha^{4},
$$

in agreement with $(5.8), 5.9$.

As discussed in [18], Chapters 9 and 10, the connection to the $\delta$-Bose gas was used to support the scaling exponent $1 / 3$ and to study the right tail of the height distribution. We added here the computation of the exact prefactor $4 / 3$ for the stretched exponential. Note that the left hand tail of the Tracy-Widom density decays as $\exp \left[-|u|^{3} / 12\right]$.

\section{$6 \quad$ Numerical evaluations}

According to 4.17 the one-point height statistics is governed by the probability density

$$
\rho_{t}(s)=\frac{\mathrm{d}}{\mathrm{d} s} F_{t}(s)=\int_{-\infty}^{\infty} \gamma_{t} \mathrm{e}^{\gamma_{t}(s-u)} \exp \left[-\mathrm{e}^{\gamma_{t}(s-u)}\right] g_{t}(u) \mathrm{d} u
$$


where $g_{t}(u)$ is defined in (3.14). This expression is sufficiently concrete to allow for some exploratory numerical studies. In Appendix A we establish that $g_{t}(s)$ can be approximated for large $t$ as

$$
g_{t}(s)=\rho_{\mathrm{TW}}(s)+\widetilde{g}_{t}(s)+\mathcal{O}\left(\gamma_{t}^{-6}\right)
$$

with the first order correction $\widetilde{g}_{t}(s)$ given in A.17). Correspondingly one has the first order approximation to $\rho_{t}(s)$ as

$$
\rho_{t}^{(1)}(s)=\int \gamma_{t} \mathrm{e}^{\gamma_{t}(s-u)} \exp \left[-\mathrm{e}^{\gamma_{t}(s-u)}\right]\left(\rho_{\mathrm{TW}}(u)+\widetilde{g}_{t}(u)\right) \mathrm{d} u .
$$

$\widetilde{g}_{t}$ can be expressed through quantities related to the Tracy-Widom distribution, see (A.30), and tabulated in 20]. The evaluation is displayed in Fig. 1, where the vertical axis corresponds to $\gamma_{t}=1$. In Fig. 2 we plot $\rho_{t}^{(1)}$ as defined in 6.3. $\rho_{t}^{(1)}$ turns negative at $\gamma_{t} \cong 5$, which signals the border of validity of the first order approximation. As shown in Fig. 3, at $\gamma_{t}=2$ the difference $\rho_{t}^{(1)}-\rho_{\text {Tw }}$ has two nodes while the difference $\rho_{t}-\rho_{\mathrm{TW}}$ as displayed in Fig. 5 below has only a single node, again indicating qualitative differences.

A more demanding approach is a numerical evaluation of the determinants defining $g_{t}$. For this purpose the kernels of $B_{t}$ and $P_{\mathrm{Ai}}$ are computed using Mathematica. One chooses a properly adjusted equally spaced grid of 120 points for each variable resulting in a matrix $\left\{M_{i j}\right\}_{i, j=1, \ldots, 120}$. The step size for $s$ is 0.1 covering the interval $[-6,6]$. The projection $P_{s}$ means to restrict $M$ to $i, j=(60+10 s), \ldots, 120$. The respective determinant is then evaluated for various $s, t$ again using Mathematica. To reach higher precision and earlier times it would be advisable to use the more refined methods of [21].

$\rho_{t}$ is displayed for $\gamma_{t}=2,5,10$ in Fig. 4. The dotted line is the limiting TracyWidom density. For better visibility, in Fig. 5 we plot the difference $\rho_{t}(s)-\rho_{\mathrm{TW}}(s)$ at the same times. In Fig. 6 we test directly the precision of the first order correction by plotting $\widetilde{g}_{t}$ at $\gamma_{t}=10$ and $g_{t}-\rho_{\mathrm{TW}}(s)$. While there is qualitative agreement we would have expected a much smaller error, in particular at the left minimum. A much cruder comparison is to plot merely the first moments of $\rho_{t}$ and $\rho_{t}^{(1)}$. As displayed in Fig. 7 the agreement is fairly precise beyond $\gamma_{t}=5$.

As argued in the Introduction, features of our exact solution should be visible also in the corner growth model with finer and finer details being revealed as $q$ is decreased from 1 to $\frac{1}{2}+\beta \sqrt{\varepsilon}$. To support this claim we carried out a few Monte Carlo simulations. Time steps have a sufficiently fine discretization and random sequential updating is used. We measure the total number of signed jumps between sites 0 and 1 . For $q=1$ at $t=10^{3} \mathrm{MC}$ steps the height distribution has an effective range of 30 equally spaced points. Except for the shifted mean, the Tracy-Widom density is an accurate approximation [3]. For earlier times the height takes so few values that correction to $\rho_{\mathrm{TW}}$ does not seem to be a meaningful notion. In Fig. 8 we plot the corresponding result for $q=0.6$ at $t=1024 \mathrm{MC}$ steps with an average 
over $10^{4}$ realizations. We compare with the exact solution at $\gamma_{t}=0.94$. Clearly, this is a better approximation than the Tracy-Widom distribution indicated by the dashed line.

Acknowledgements. We are grateful to Michael Prähofer for many illuminating discussions. H. S. thanks Jeremy Quastel for emphasizing the importance of the crossover WASEP. This work is supported by a DFG grant. In addition T.S. acknowledges the support from KAKENHI (9740044) and H.S. from Math-for-Industry of Kyushu University.

\section{A Appendix: Expansion in $1 / t$}

Expansion of the determinants. We follow as closely as possible the notation in [19], simply referred to as TW. As only difference we set

$$
\psi(x)=\operatorname{Ai}(x) \quad(A(x) \text { in } \mathrm{TW})
$$

and

$$
\psi_{\lambda}(x)=\psi(x+\lambda), \quad \psi_{0}(x)=\psi(x) .
$$

We define the operator

$$
B_{t}=K+C_{t}
$$

with the kernels

$$
K(x, y)=\int_{0}^{\infty} \mathrm{d} \lambda \psi_{\lambda}(x) \psi_{\lambda}(y)
$$

formerly denoted by $K_{\mathrm{Ai}}$, and

$$
C_{t}(x, y)=\int_{0}^{\infty} \mathrm{d} \lambda\left(\mathrm{e}^{\gamma_{t} \lambda}-1\right)^{-1}\left(\psi_{\lambda}(x) \psi_{\lambda}(y)-\psi_{-\lambda}(x) \psi_{-\lambda}(y)\right)
$$

and the unnormalized projection $P_{\psi}$ with kernel $\psi(x) \psi(y)$.

We consider the difference

$$
g_{t}(s)=\operatorname{det}\left(1-K-C_{t}+P_{\psi}\right)-\operatorname{det}\left(1-K-C_{t}\right) .
$$

The projection $P_{s}$ in $(3.14)$ is taken into account by defining the determinant in $L^{2}([s, \infty))$ with scalar product $\langle\cdot, \cdot\rangle$. For fixed $s$ we want to expand $g_{t}(s)$ in $\gamma_{t}^{-1}$, which means to regard $C_{t}$ as a small perturbation.

One starts from

$$
\begin{aligned}
g_{t} & =\operatorname{det}\left(1-K-C_{t}\right)\left\langle\psi,\left(1-K-C_{t}\right)^{-1} \psi\right\rangle \\
& =\operatorname{det}(1-K) \operatorname{det}\left(1-(1-K)^{-1} C_{t}\right)\left\langle\psi,\left(1-K-C_{t}\right)^{-1} \psi\right\rangle,
\end{aligned}
$$


expands the Fredholm determinant as

$$
\operatorname{det}\left(1-(1-K)^{-1} C_{t}\right)=1-\operatorname{tr}\left((1-K)^{-1} C_{t}\right)+\mathcal{O}\left(C_{t}^{2}\right),
$$

and writes the resolvent as

$$
\left\langle\psi,\left(1-K-C_{t}\right)^{-1} \psi\right\rangle=\left\langle\psi,(1-K)^{-1} \psi\right\rangle+\left\langle\psi,(1-K)^{-1} C_{t}(1-K)^{-1} \psi\right\rangle+\mathcal{O}\left(C_{t}^{2}\right) .
$$

The leading term equals $F^{\prime} / F$, see [12], where

$$
F=F_{\mathrm{TW}}(s)=\operatorname{det}\left(1-P_{s} K_{\mathrm{Ai}} P_{s}\right)=\operatorname{det}(1-K) .
$$

The term linear in $C_{t}$ is

$$
\begin{aligned}
\widetilde{g}_{t} & =F\left\{\left\langle\psi,(1-K)^{-1} C_{t}(1-K)^{-1} \psi\right\rangle-\left\langle\psi,(1-K)^{-1} \psi\right\rangle \operatorname{tr}\left((1-K)^{-1} C_{t}\right)\right\} \\
& =g_{t}-F^{\prime}+\mathcal{O}\left(C_{t}^{2}\right) .
\end{aligned}
$$

Inserting the definition of $C_{t}$ from (A.5), one arrives at

$$
\widetilde{g}_{t}=F \int_{0}^{\infty} \mathrm{d} \lambda\left(\mathrm{e}^{\gamma t \lambda}-1\right)^{-1}(G(\lambda)-G(-\lambda))
$$

with

$$
G(\lambda)=\left\langle\psi,(1-K)^{-1} \psi_{\lambda}\right\rangle\left\langle\psi_{\lambda},(1-K)^{-1} \psi\right\rangle-\left\langle\psi_{\lambda},(1-K)^{-1} \psi_{\lambda}\right\rangle\left\langle\psi,(1-K)^{-1} \psi\right\rangle .
$$

Rescaling by $\gamma_{t}$ one arrives at

$$
\widetilde{g}_{t}=F \gamma_{t}^{-1} \int_{0}^{\infty} \mathrm{d} \lambda\left(\mathrm{e}^{\lambda}-1\right)^{-1}\left(G\left(\lambda / \gamma_{t}\right)-G\left(-\lambda / \gamma_{t}\right)\right) .
$$

Thus the expansion in $1 / t$ amounts to a Taylor expansion of $\widetilde{G}(\lambda)=G(\lambda)-G(-\lambda)$ at $\lambda=0$.

One has $\widetilde{G}(0)=0, \widetilde{G}^{\prime \prime}(0)=0$, since $\widetilde{G}$ is odd. Furthermore $\widetilde{G}^{\prime}(0)=0$ and

$$
\begin{aligned}
\widetilde{G}^{\prime \prime \prime}(0)= & 6\left(\left\langle\psi^{\prime \prime},(1-K)^{-1} \psi\right\rangle\left\langle\psi^{\prime},(1-K)^{-1} \psi\right\rangle\right. \\
& \left.-\left\langle\psi^{\prime \prime},(1-K)^{-1} \psi^{\prime}\right\rangle\left\langle\psi,(1-K)^{-1} \psi\right\rangle\right) .
\end{aligned}
$$

Hence, using that

$$
\int_{0}^{\infty} \lambda^{3}\left(\mathrm{e}^{\lambda}-1\right)^{-1} \mathrm{~d} \lambda=\pi^{4} / 15
$$

it holds

$$
\begin{aligned}
& \widetilde{g}_{t}=2 \gamma_{t}^{-4}\left(\pi^{4} / 15\right) F\left(\left\langle\psi^{\prime \prime},(1-K)^{-1} \psi\right\rangle\left\langle\psi^{\prime},(1-K)^{-1} \psi\right\rangle\right. \\
&\left.-\left\langle\psi^{\prime \prime},(1-K)^{-1} \psi^{\prime}\right\rangle\left\langle\psi,(1-K)^{-1} \psi\right\rangle\right) .
\end{aligned}
$$


Since $\widetilde{g}_{t}$ is of order $\gamma_{t}^{-4}$, in principle, the terms of $\mathcal{O}\left(C_{t}^{2}\right)$ could yield a more slowly decaying contribution. By expanding to second order, one checks that this contribution is at least of order $\gamma_{t}^{-5}$. Thus A.17 is the leading order in the $1 / t$ expansion.

Link to the Tracy-Widom distribution. For a numerical computation of $\widetilde{g}_{t}$ it is convenient to use the well tabulated $F[20]$. One introduces, see TW,

$$
\begin{aligned}
Q & =(1-K)^{-1} \psi, \quad P=(1-K)^{-1} \psi^{\prime}, \\
q(s) & =Q(s), p(s)=P(s), u(s)=\langle\psi, Q\rangle, v(s)=\langle\psi, P\rangle .
\end{aligned}
$$

To compute A.17) one still needs $\left\langle\psi^{\prime \prime}, Q\right\rangle$ and $\left\langle\psi^{\prime \prime}, P\right\rangle$.

(i) Computation of $\left\langle\psi^{\prime \prime}, P\right\rangle$. We claim that

$$
\left\langle\psi^{\prime \prime}, P\right\rangle=\frac{1}{2}\left(v^{2}-p^{2}\right)
$$

Proof: One starts from TW, below (2.14),

$$
\left\langle\psi^{\prime}, P^{\prime}\right\rangle=\left\langle\psi^{\prime},(1-K)^{-1} \psi^{\prime \prime}\right\rangle+\left\langle\psi^{\prime},\left[D,(1-K)^{-1}\right] \psi^{\prime}\right\rangle .
$$

On the other hand

$$
\left\langle\psi^{\prime}, P^{\prime}\right\rangle=-\psi^{\prime}(s) P(s)-\left\langle\psi^{\prime \prime}, P\right\rangle .
$$

Hence

$$
-2\left\langle\psi^{\prime \prime},(1-K)^{-1} \psi^{\prime}\right\rangle=\psi^{\prime}(s) p+\left\langle\psi^{\prime},\left[D,(1-K)^{-1}\right] \psi^{\prime}\right\rangle .
$$

From TW (2.13) and the definitions of $R$ and $\rho$ there,

$$
\left\langle\psi^{\prime},\left[D,(1-K)^{-1}\right] \psi^{\prime}\right\rangle=-v^{2}+\left\langle\psi^{\prime}, R\right\rangle\left\langle\psi^{\prime}, \rho\right\rangle
$$

and from TW above (2.18),

$$
\left\langle\psi^{\prime}, R\right\rangle=-\left(\psi^{\prime}(s)-p\right), \quad\left\langle\psi^{\prime}, \rho\right\rangle=p,
$$

which establishes the claim.

(ii) Computation of $\left\langle\psi^{\prime \prime}, Q\right\rangle$. We claim that

$$
\left\langle\psi^{\prime \prime}, Q\right\rangle=-\int_{s}^{\infty} p\left(s^{\prime}\right)^{2} \mathrm{~d} s^{\prime}+v u-q p .
$$

Proof: Using TW (2.14) one starts from

$$
\begin{aligned}
\left\langle\psi^{\prime}, Q^{\prime}\right\rangle & =-\psi^{\prime}(s) q-\left\langle\psi^{\prime \prime}, Q\right\rangle \\
& =\left\langle\psi^{\prime}, P\right\rangle-\left\langle\psi^{\prime}, Q\right\rangle u+q\left\langle R, \psi^{\prime}\right\rangle \\
& =\left\langle\psi^{\prime}, P\right\rangle-v u+q\left(p-\psi^{\prime}(s)\right) .
\end{aligned}
$$


Hence

$$
\left\langle\psi^{\prime \prime}, Q\right\rangle=-\left\langle\psi^{\prime}, P\right\rangle+v u-q p
$$

Next we consider

$$
\begin{aligned}
\frac{d}{d s}\left\langle\psi^{\prime}, P\right\rangle & =-\psi^{\prime}(s) p-\left\langle\psi^{\prime}, R\right\rangle p \\
& =-\psi^{\prime}(s) p-\left(-\psi^{\prime}(s)+p\right) p=-p^{2}
\end{aligned}
$$

which establishes the claim.

Using the identities from TW, it holds

$$
u=F^{\prime} / F, \quad v=F^{\prime \prime} / 2 F, \quad q^{2}=u^{2}-2 v, \quad p=q^{\prime}+q u .
$$

In combination with A.17, A.18, A.19, and A.25, one arrives at

$$
\begin{aligned}
\widetilde{g}_{t}(s)= & \left(\pi^{4} / 15\right) \gamma_{t}^{-4} F(s) \\
& \times\left(-2 v(s)\left(q(s) p(s)+\int_{s}^{\infty} p\left(s^{\prime}\right)^{2} \mathrm{~d} s^{\prime}\right)+u(s)\left(v(s)^{2}+p(s)^{2}\right)\right),
\end{aligned}
$$

which is the starting point for numerical evaluations. In our notation, [20] tabulates $F, q, q^{\prime}, u$. From A.29 we then deduce $p$ and $v$ and only the computation of the integral remains.

\section{References}

[1] M. Kardar, G. Parisi, Y-C. Zhang, Dynamic scaling of growing interfaces, Phys. Rev. Lett. 56, 889-892 (1986).

[2] M. Balázs, J. Quastel, T. Seppäläinen, Scaling exponent for the Cole-Hopf solution of the KPZ/stochastic Burgers, arXiv:0909.4816, preprint (2010).

[3] T. Sasamoto, H. Spohn, Universality of the one-dimensional KPZ equation, arXiv, preprint (2010).

[4] C.A. Tracy, H. Widom, Asymptotics in ASEP with step initial condition, Comm. Math. Phys. 290, 129-154 (2009).

[5] C.A. Tracy, H. Widom, Total current fluctuations in the asymmetric simple exclusion model, J. Math. Phys. 50, 095204 (2009).

[6] K. Johansson, Shape fluctuations and random matrices, Comm. Math. Phys. 209, 437-476 (2000).

[7] M. Prähofer, private communication, 2009. 
[8] C. A. Tracy, H. Widom, Formulas for ASEP with two-sided Bernoulli initial condition, arXiv: 1001.4766, preprint (2010).

[9] L. Bertini, G. Giacomin, Stochastic Burgers and KPZ equations from particle systems, Comm. Math. Phys. 183, 571-607 (1997).

[10] J. Glimm, A. Jaffe, Quantum Physics: A Functional Integral Point of View. Springer-Verlag, Berlin, 1987.

[11] A. De Masi, E. Presutti, E. Scacciatelli, The weakly asymmetric exclusion process, Ann. Inst. Henri Poincaré, Probab. et Stat. 25, 1-38 (1989).

[12] T. Sasamoto, H. Spohn, The crossover regime for the weakly asymmetric simple exclusion process, arXiv, preprint (2010).

[13] J. Gärtner, Convergence towards Burgers equation and propagation of chaos for weakly asymmetric exclusion process. Stoch. Processes Appl. 27, 233-260 (1988).

[14] P. Dittrich, J. Gärtner, A central limit theorem for the weakly asymmetric simple exclusion process, Math. Nachr. 151, 75-93 (1991).

[15] G.M. Schütz, Duality relations for asymmetric exclusion processes, J. Stat. Phys. 86, 1265-1288 (1997).

[16] J.B. McGuire, Study of exactly soluble one-dimensional $N$-body problems, J. Math. Phys. 5, 622-636 (1964).

[17] M. Prähofer, H. Spohn, Universal distributions for growth processes in $1+1$ dimensions and random matrices, Phys. Rev. Lett. 84, 4882-4885 (2000).

[18] M. Kardar, Statistical Mechanics of Fields, Cambridge University Press, 2007.

[19] C.A. Tracy, H. Widom, Level-spacing distributions and the Airy kernel, Commun. Math. Phys. 159, 151-174 (1994).

[20] M. Prähofer, homepage, M5, Zentrum Mathematik, TU München.

[21] F. Bornemann, On the numerical evaluation of Fredholm determinants, Math. Comp., online (2009). 


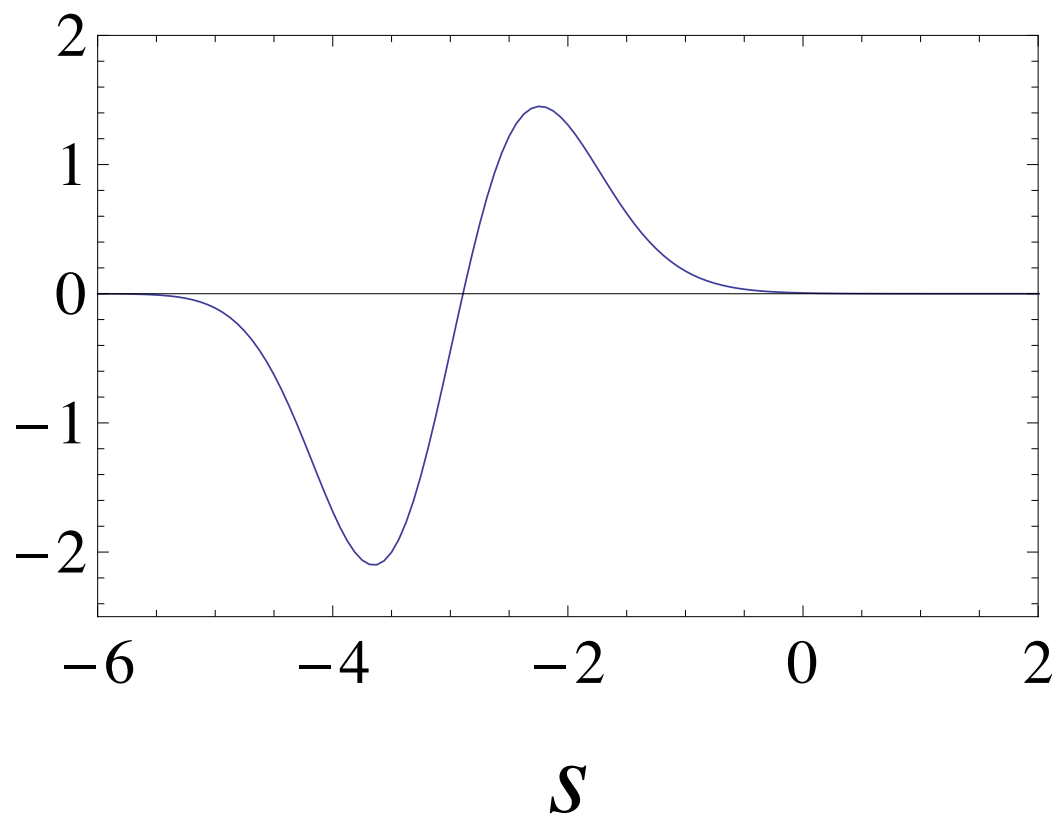

Figure 1: The first order correction $\tilde{g}_{t}(s)$ in (6.2). The vertical scale is for $\gamma_{t}=1$.

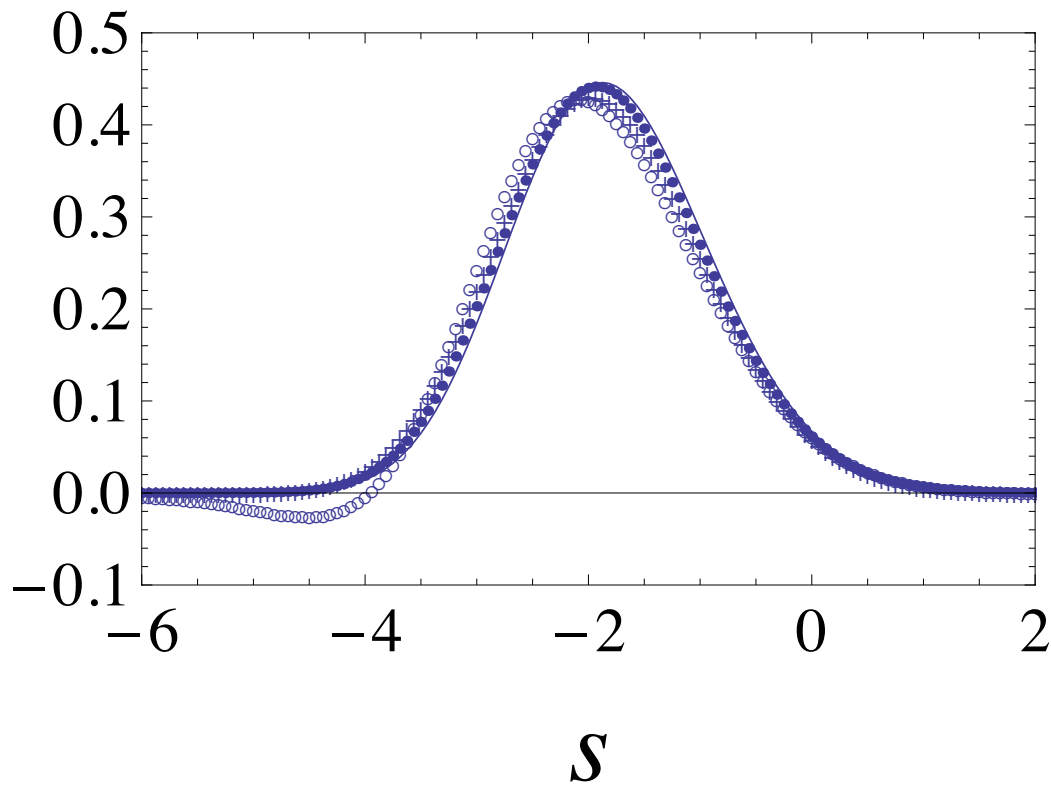

Figure 2: The first order approximation $\rho_{t}^{(1)}(s)$ in 6.3 for $\gamma_{t}=2(\circ), 5(+), 10(\bullet)$. 


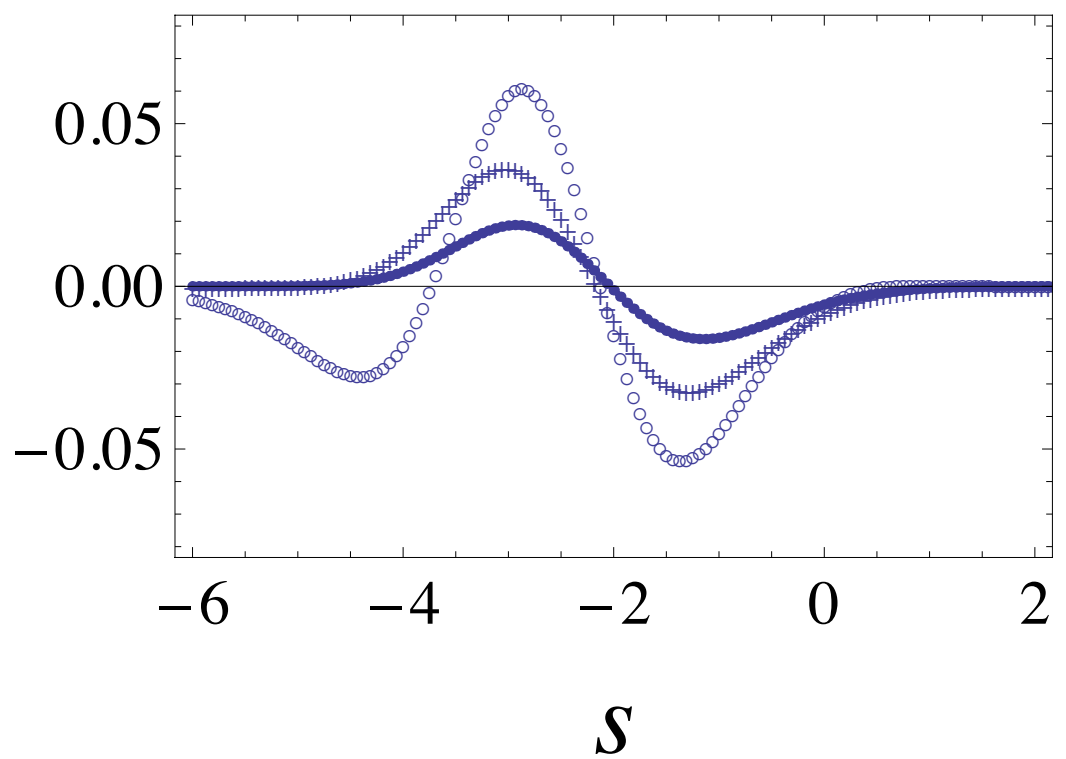

Figure 3: The difference $\rho_{t}^{(1)}(s)-\rho_{\mathrm{TW}}(s)$ for $\gamma_{t}=2(\circ), 5(+), 10(\bullet)$.

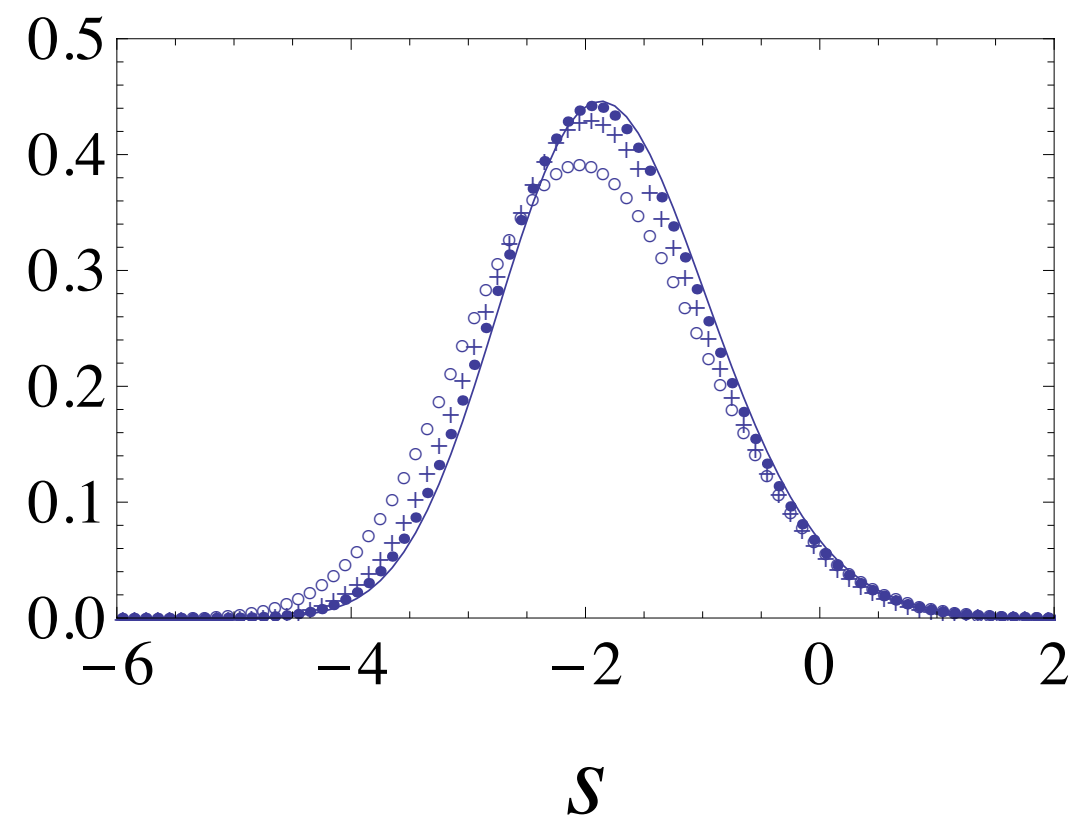

Figure 4: KPZ probability densities $\rho_{t}(s)$ for $\gamma_{t}=2(\circ), 5(+), 10(\bullet)$ based on the evaluation of the determinants. 


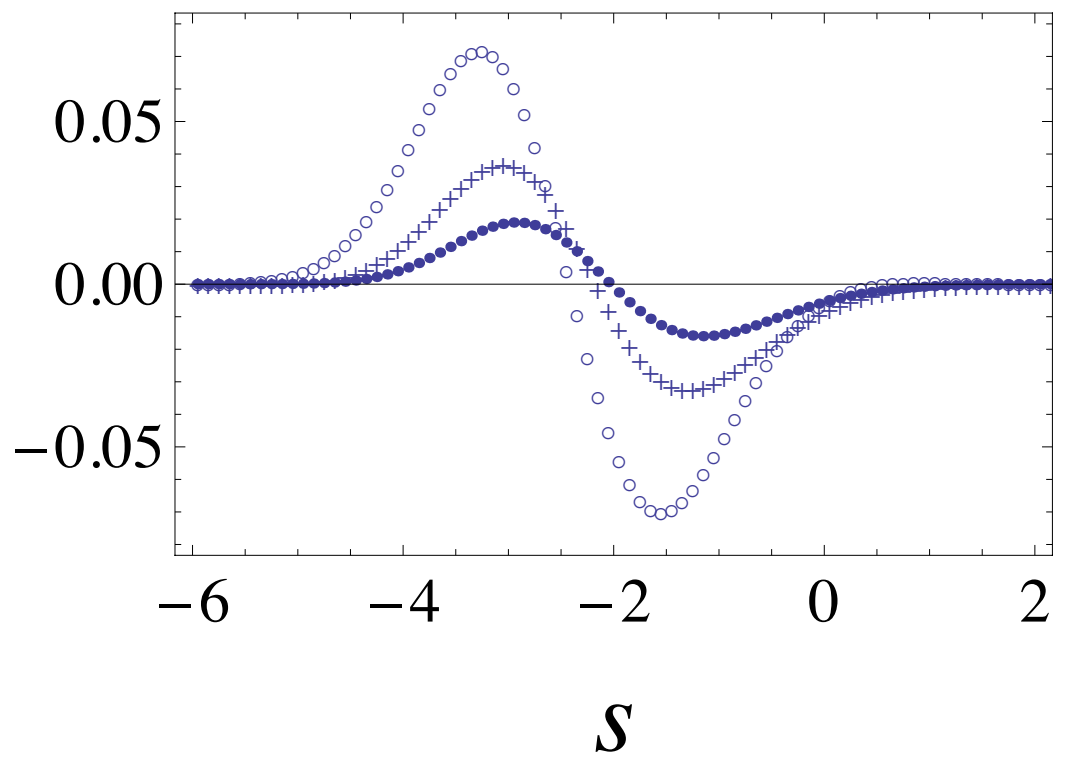

Figure 5: The difference $\rho_{t}(s)-\rho_{\mathrm{TW}}(s)$ for $\gamma_{t}=2(\mathrm{o}), 5(+), 10(\bullet)$ based on the evaluation of the determinants.

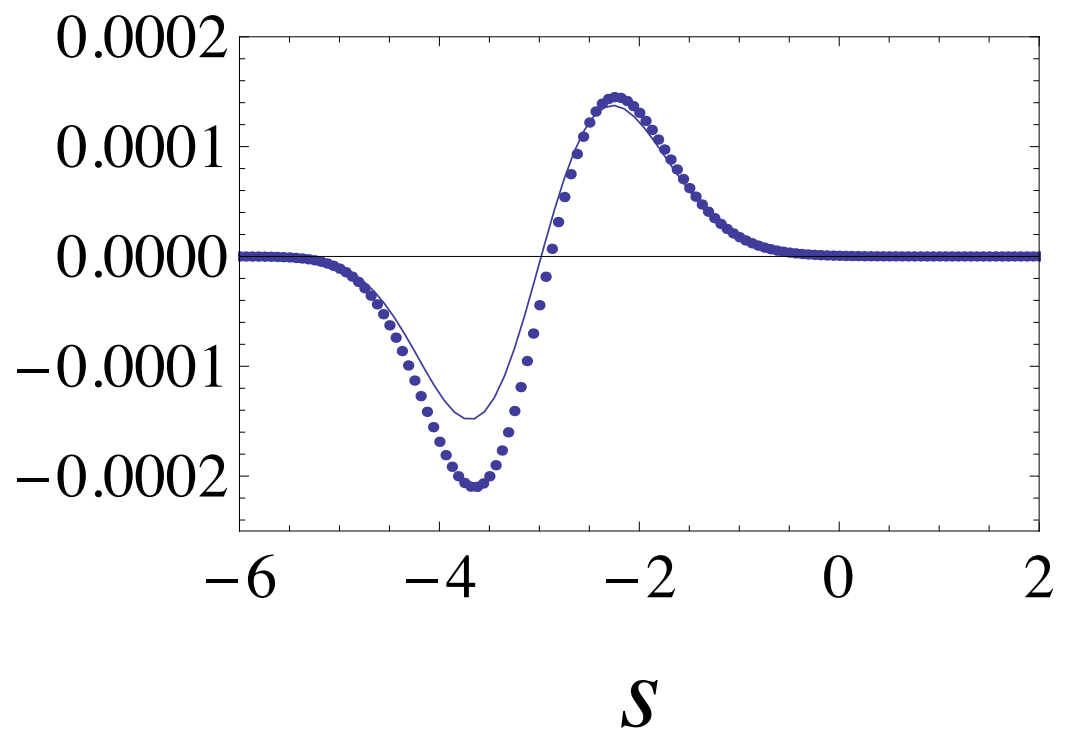

Figure 6: $\tilde{g}_{t}(s)(-)$ and $g_{t}(s)-\rho_{\mathrm{TW}}(s)(\bullet)$ for $\gamma_{t}=10$. 


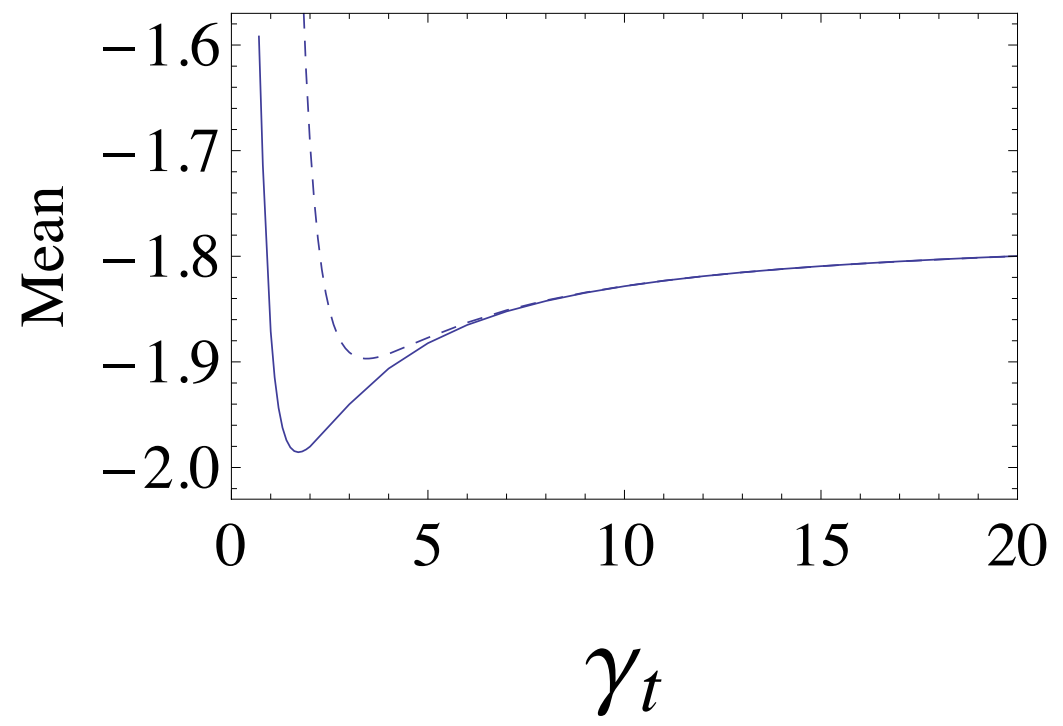

Figure 7: First moment of $\rho_{t}(-)$ and $\rho_{t}^{(1)}(--)$ as a function of $\gamma_{t}$.

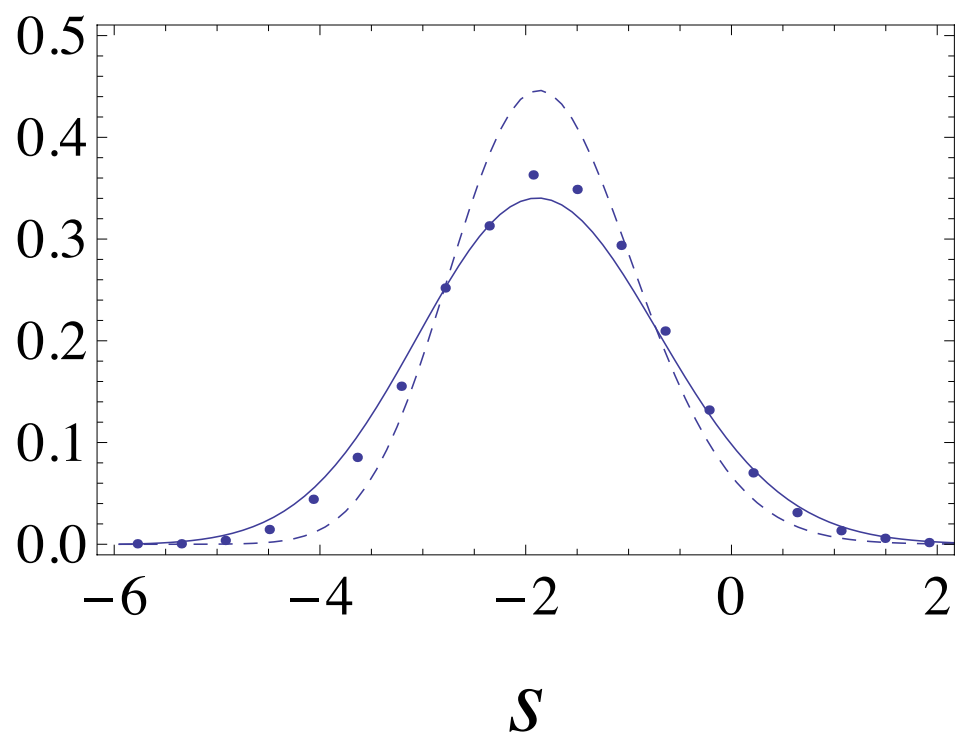

Figure 8: The exact KPZ density at $\gamma_{t}=0.94(-)$ and the PASEP Monte Carlo at $q=0.6, t=1024 \mathrm{MC}$ steps $(\bullet)$. The dashed line is the Tracy-Widom density. 